\title{
Web monitoring of EOS front-end ground operations, science downlinks and level 0 processing
}

\author{
Guy R. Cordier ${ }^{1}$ \\ Lockheed Martin Mission Systems / GSFC, Greenbelt, MD 20771, USA \\ Chris Wilkinson ${ }^{2}$ \\ NASA GSFC, Greenbelt, MD 20771, USA \\ and \\ Bruce McLemore ${ }^{3}$ \\ Honeywell TSI / GSFC, Greenbelt, MD 20771, USA
}

\begin{abstract}
This paper addresses the efforts undertaken and the technology deployed to aggregate and distribute the metadata characterizing the real-time operations associated with NASA Earth Observing Systems (EOS) high-rate front-end systems and the science data collected at multiple ground stations and forwarded to the Goddard Space Flight Center for level 0 processing. Station operators, mission project management personnel, spacecraft flight operations personnel and data end-users for various EOS missions can retrieve the information at any time from any location having access to the internet. The users are distributed and the EOS systems are distributed but the centralized metadata accessed via an external web server provide an effective global and detailed view of the enterprise-wide events as they are happening. The data-driven architecture and the implementation of applied middleware technology, open source database, open source monitoring tools, and external web server converge nicely to fulfill the various needs of the enterprise. The timeliness and content of the information provided are key to making timely and correct decisions which reduce project risk and enhance overall customer satisfaction. The authors discuss security measures employed to limit access of data to authorized users only.
\end{abstract}

\section{Introduction}

$\mathrm{N}$ ASA EOS Data and Operations System (EDOS) reengineering includes the central control and monitoring system $(M \& C)$ to support daily operations using web browsers technologies. The objectives are driven by the need to streamline operations for costs reduction, to better enable the system users make timely decisions based on near-realtime information (meta data) available via external web servers and to facilitate new mission insertion into EDOS.

This effort follows on previous major reengineering initiatives ${ }^{1,2}$ successfully applied to the system in the 2006 time frame. EDOS deployed a data-driven solution to capture the science downlinks at the ground stations. The solution eliminated routine operator setup control functions associated with the front-end devices for scheduled mission support. EDOS is now driven by identifying the incoming spacecraft ID within the frame of the incoming serial data/clock signal and no schedules. This aspect of the system was discussed in a previous paper by the authors presented at the SpaceOps conference in Rome in 2006. The automation of the data capture process has been exceptionally reliable ever since.

Still, with the benefits of the automated tasks executed across the enterprise moving data from the capture site through the Level Zero processing Facility (LZPF) and onward to the end-users facilities, Operations personnel have to rely on a robust control and monitor system to provide a centralized view of systems heath status and of the

\footnotetext{
${ }^{1}$ System Engineer, Sr. Staff, GSFC/Code 423, Greenbelt, MD 20771

${ }^{2}$ ESMO Deputy Manager, GSFC/Code 428, Greenbelt, MD 20771.

${ }^{3}$ MOMS EDOS Project Manager, GSFC/ Code 428, Greenbelt, MD 20771 
processing state of the data at any given time. The availability in near-realtime of the science downlinks data quality statistics, for example, enables the flight operations teams (FOT), ground station operators to make timely decisions involving ground station reconfigurations and/or on-board spacecraft recorder management decisions when anomalous situations develop

\section{Background}

The X-Band data are downlinked at Svalbard, Norway, at four possible antennas SG-1, SG-2, SG-3, and SG-4; at Fairbanks, Alaska - AGS, PF-1, PF-2, GL-A, GL-B, and GL-C; at White Sands, New Mexico - STGT and WSGT; and at one antenna at the Wallops Flight Facility (WFF) in Virginia. The 13 antennas are shared with other customers and programs. Figure 1 depicts the functional configuration of the system C5.1 release currently undergoing acceptance testing at the time of this writing (April 2008). EDOS has one EBox - Sender (S) dedicated to each antenna to service the data capture in a data-driven mode of operation 24X7. The EBox-S forwards the data across a high rate network to the EBox - Receiver (R) at the LZPF located at GSFC.

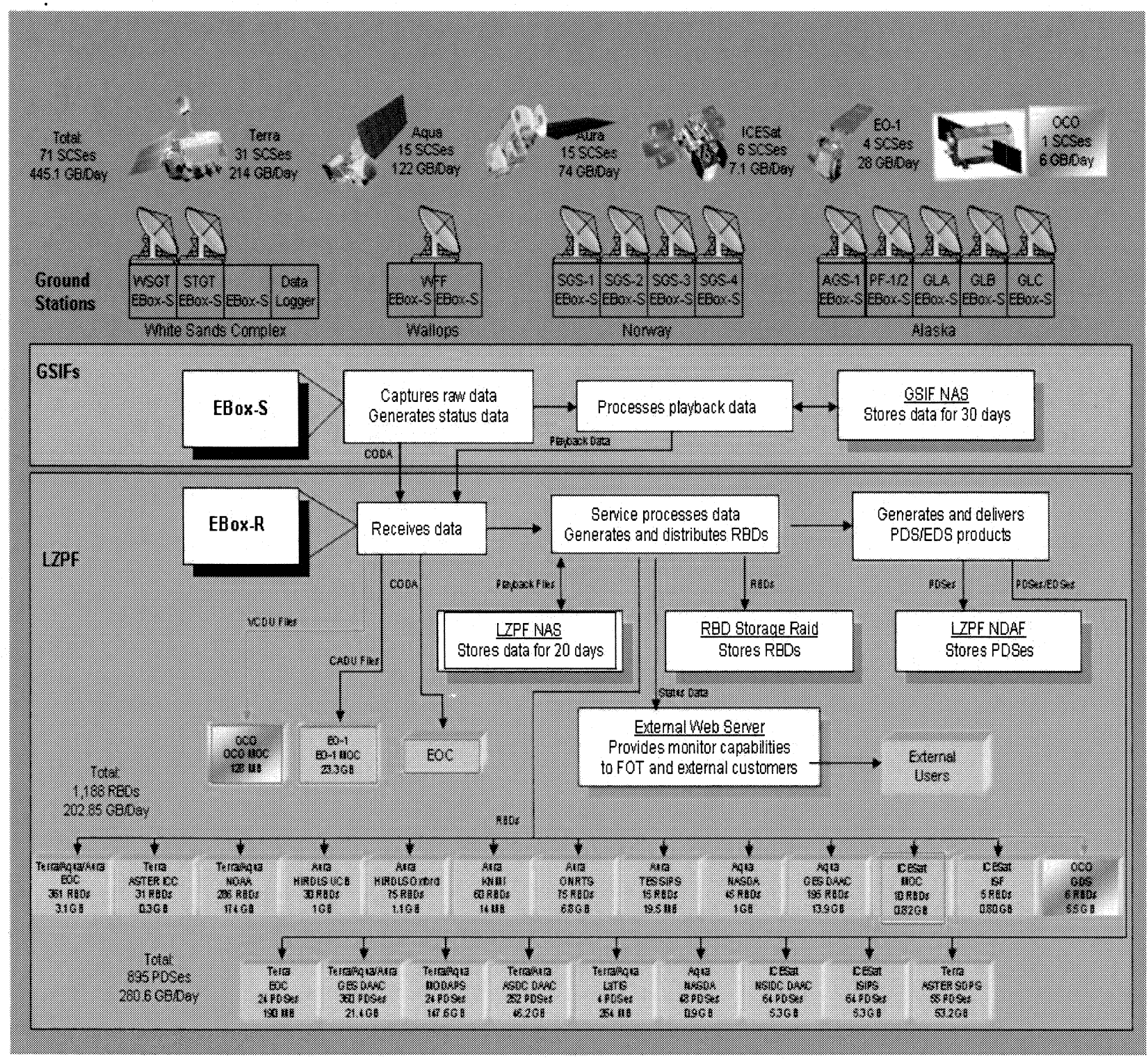

Figure 1. EDOS Distributed Functions and Data Flows 
The LZPF primary function is to perform the level zero processing on the data received from the ground stations, per the mission requirements, and to generate, deliver and archive the products. The centralization of the processing permits the creation of data sets of contiguous data, made from data segments downlinked from the EOS spacecraft at the various sites. Fig 1 depicts also the products types and quantity of data delivered daily to the various external organizations. Also shown are the four control centers involved with spacecraft mission control, the EOS Control Center (EOC) at GSFC, the EO-1 Mission Operations Control (MOC) at GSFC, the Mission Operations Center (MOC) for ICESat at the University of Colorado and the Mission Operations Control (MOC) for the Orbiting Carbon Observatory (OCO).

\section{EDOS Centralized Monitor and Control (M\&C) System}

The reengineering of the EDOS M\&C System takes into account users past experience with the current Computer Human Interface (CHI) used by EDOS for the last decade, new web based browser technologies, and new mission demands. To summarize, the system has to

- Provide for central control and monitoring functions associated with the EBoxes' S and R, M\&C hosts and the communications lines

- Provide status of the downlinks in near-realtime to mission control centers managing the spacecrafts

- Service the EDOS data capture elements geographically distributed at the ground stations

- Report on the status of data products creation, distribution and archiving

- Provide detailed views of the meta data needed by the system users

- Satisfy the system security requirements

To develop a reliable system responsive to the needs of the users, the EDOS development team collaborated with the team at Kongsberg Spacetec (KSPT), Tromso, Norway. KSPT was already familiar with the EDOS infrastructure, having built the data capture devices (EBox-S) and integrated the data receiving units (EBox-R) with KSPT receiving software and EDOS-ported legacy level zero processing software.

Figure 2 depicts a generic hierarchical view of the Multimission Earth Observation System (MEOS) M\&C system as applied to EDOS. The MEOS middleware (MW) developed by KSPT forms the backbone of the EDOS Monitor and Control (M\&C) System.

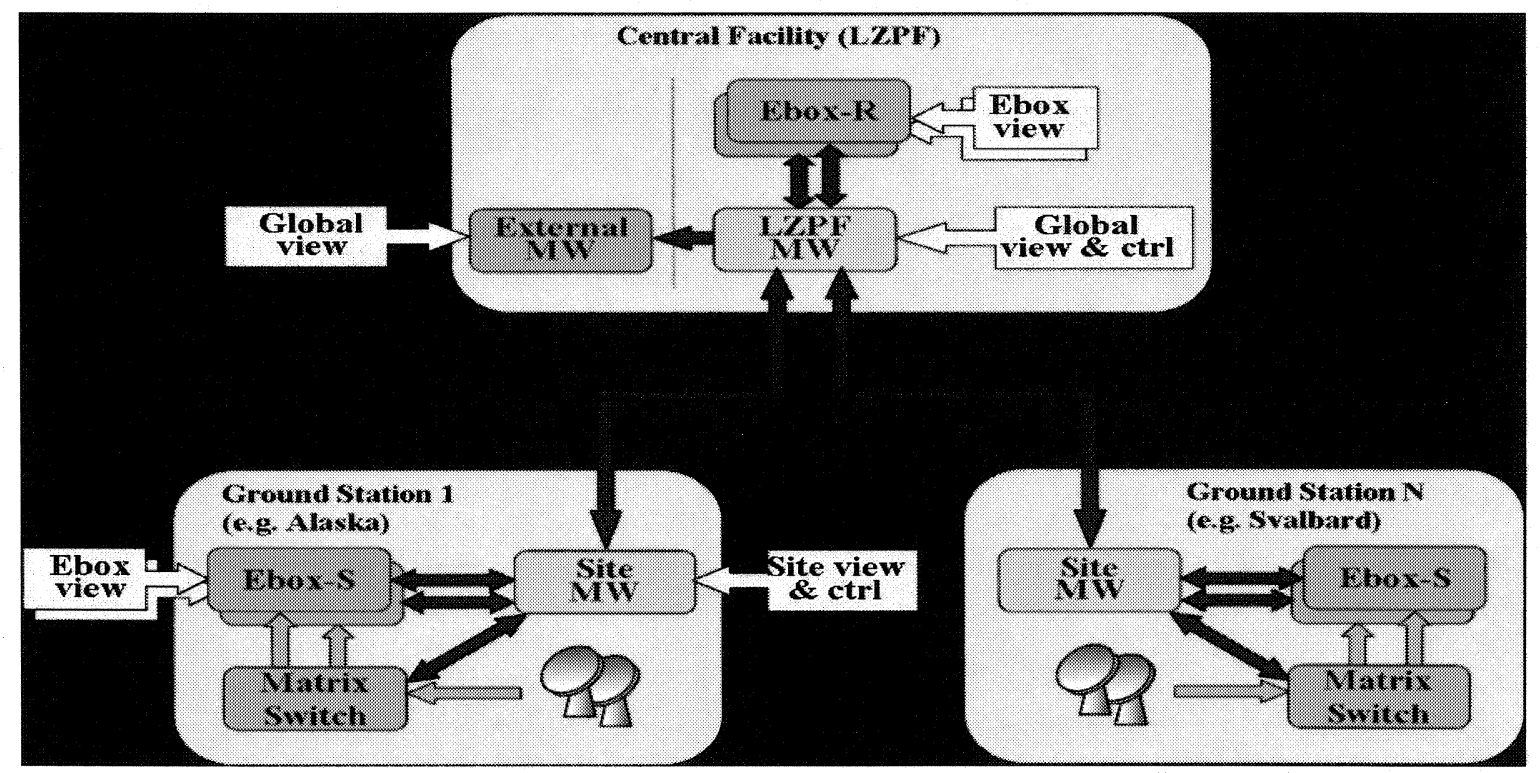

Figure 2. MEOS M\&C System applied to EDOS

The MW forms a hierarchical infrastructure for retrieving system status and for exercising control of all software components via simple, well-defined user interfaces. A MW entity resides on each EBox as well as on a dedicated machine at each site and a central one at the LZPF. Near real-time or historical system local status is available at 
each local MW instance, while the system global status is available at the LZPF MW. Each physical MW component has a hot backup for automatic failover.

The features of the EDOS MEOS M\&C System Graphical User Interfaces (GUI) include

- The principle of fire watch mode operations from a single GUI, covering the system global status

- The efficient remote as well as local access to all system components throughout the MEOS MW hierarchy

- The integrated monitor and control displays using Java 5 and Web browser, for portability and ease of maintenance

- The adaptability to support different modes of operation, and other future requirements.

The Oracle database servers (not shown on Fig 2) based GUIs provide detailed status on the EDOS data processing tasks on the EBox-R's and a long term archive of the meta data. Additional displays are available outside the MEOS GUIs to support the upcoming transition of the systems. This is merely a temporary state on how things evolved between the MEOS system applied to EDOS and the heritage data processing monitoring at EDOS back end based on an Oracle database and implemented by the EDOS development team.

\section{External Web Server}

\section{A. Architecture Overview}

The requirement on the external web server is to provide information to authorized users via the Internet and via monitor displays of the EDOS system. The appropriate security considerations must be met and the load from external user's queries on the system must not contribute in any way to degradation of the operational EDOS system performance.

An overview of the generic architecture at GSFC is represented in Figure 3 below.

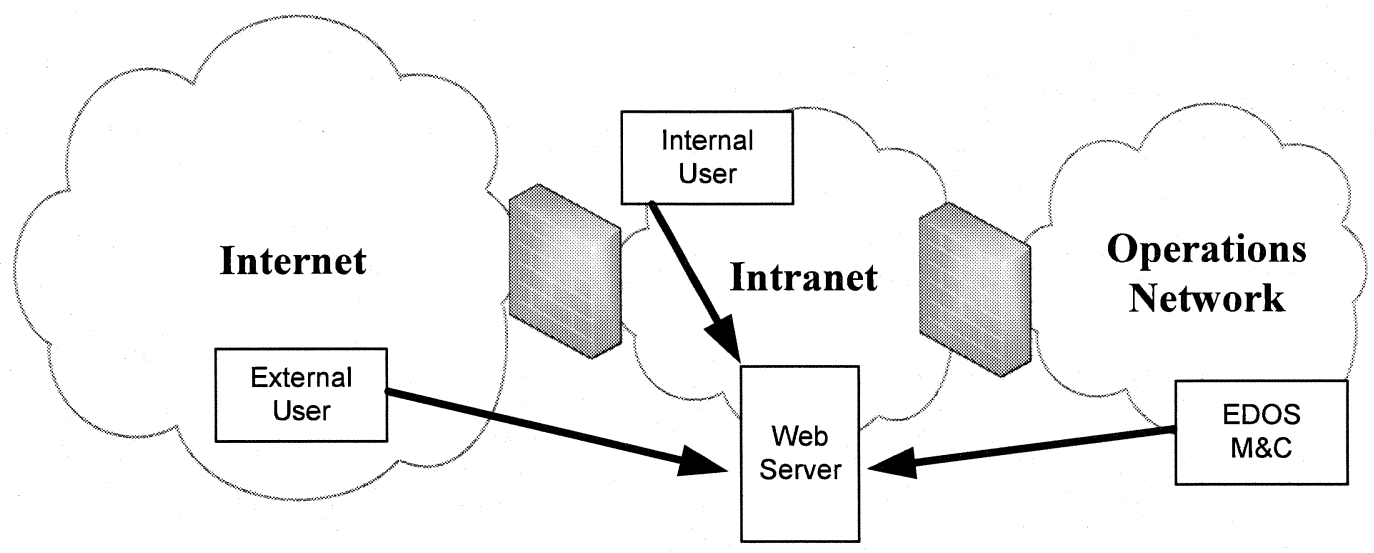

Figure 3. Generic EDOS Web Architecture at GSFC

The EBox systems are located on the closed operations network. This network can not be accessed directly from the Internet nor from the Intranet. To make the EBox systems visible to the Intranet a computer is placed on the Intranet that mirrors the content of the EBox systems. The EBox systems on the operations network push the information to the mirror on the Intranet. This communication will be unidirectional from the operations network to the Intranet. This also means that access from the Internet or Intranet is restricted to systems monitoring of mirrored data only. Executing commands or configuring units is not possible. The access from the Internet to the Intranet (and from within the Intranet) is only allowed by authorized users.

\section{B. Network / Client Configuration}

Figure 4 depicts the relationships and flows between the ground station sites (SGC, AGS, WSC, and WFF) MW boxes, the central MW boxes at the LZPF and the Client GUI on the internet. The web server (httpd) on the Intranet is used for application development and storage of the GUI layout description files.

In order for a user to monitor the systems, the following steps are required: 
1) Obtain authorization for connection to the web server

2) Start a browser, e.g. Firefox, and access the mainpage on the MW Mirror computer, which hosts the web server

3) Download the client software through Java Web Start

4) Application starts and a login is presented

5) Upon successful login, the GUI is constructed from the layout files on the web server

6) The GUI client will now receive real time status and information form the EBox systems

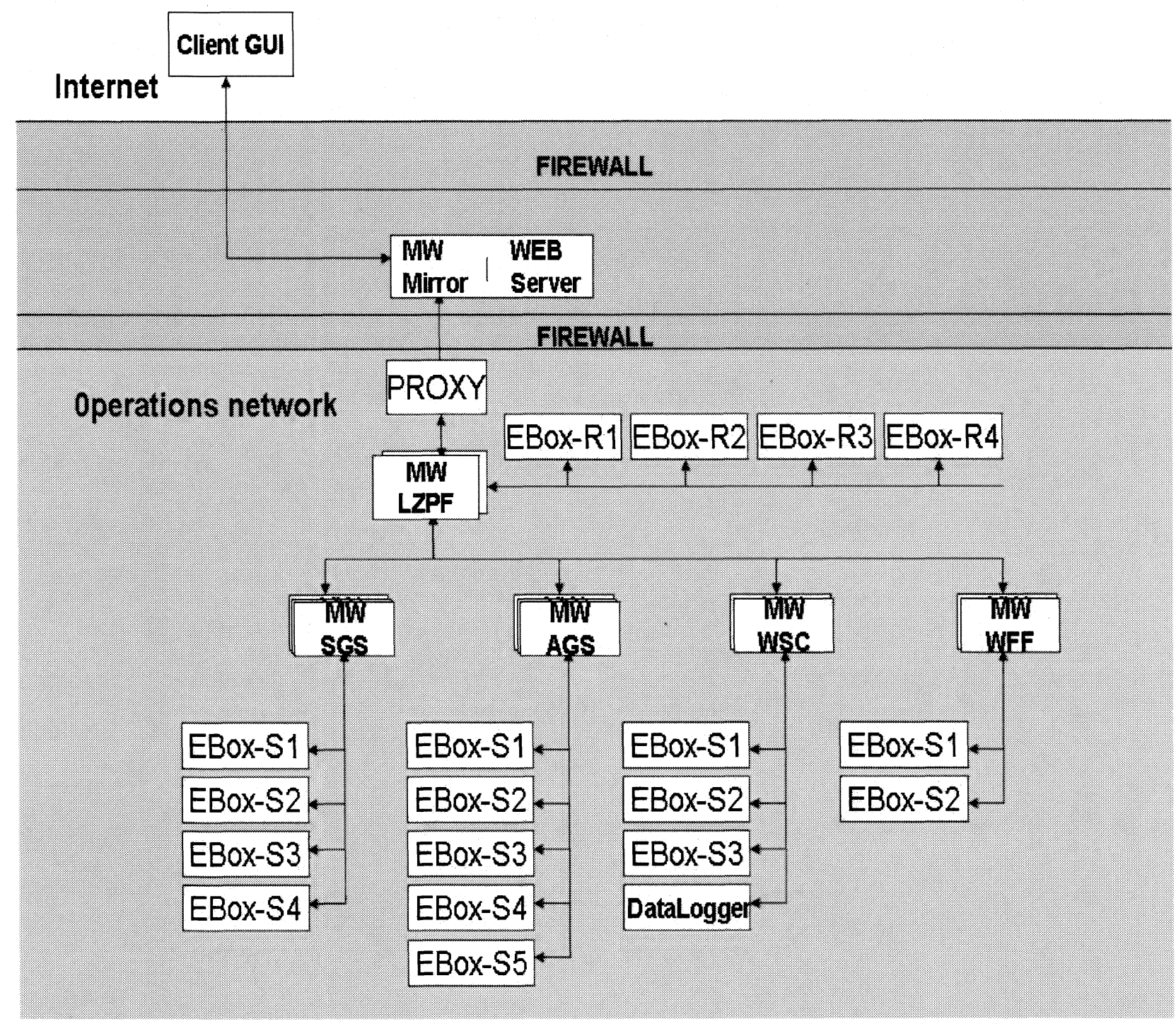

Figure 4. Detailed Network Overview

\section{Security Drivers}

The key security points can be summarized as follows:

- Only unidirectional flow of data from the Operations network to the Intranet is permitted through the Firewall

- Currently the Internet access is done through a secure channel, and the external computer and owner must have been authorized to access the Intranet

- The client software on the internet can only reach as far as the MW mirror (web server) on the Intranet. Communications from the MW Mirror to the Operations network is not possible.

- In the future the external web server will be moved to a more publicly accessible location to support the large number of external users of the EOS meta-data 


\section{GUI Views}

EDOS operation staff will use the new centralized web based GUIs within the operational (network) environment to perform the EDOS control and monitor functions. The authorized external web users use an identical monitor only view of the status of the systems and of data in near-realtime. The following figures show a selection of status GUIs. At the oral presentation of this paper, the authors will access the external server for a live presentation of the GUI views.

1. The main GUI below (Fig 5) displays a consolidated view of the ground sites and of the LZPF. A serious malfunction of a subsystem would turn the subsystem icon red and would require operator intervention. Status information on processes is collected from the systems for updates. One feature of the MW is that MW servers exchange updates between the sites MW and the central MW only upon content change.

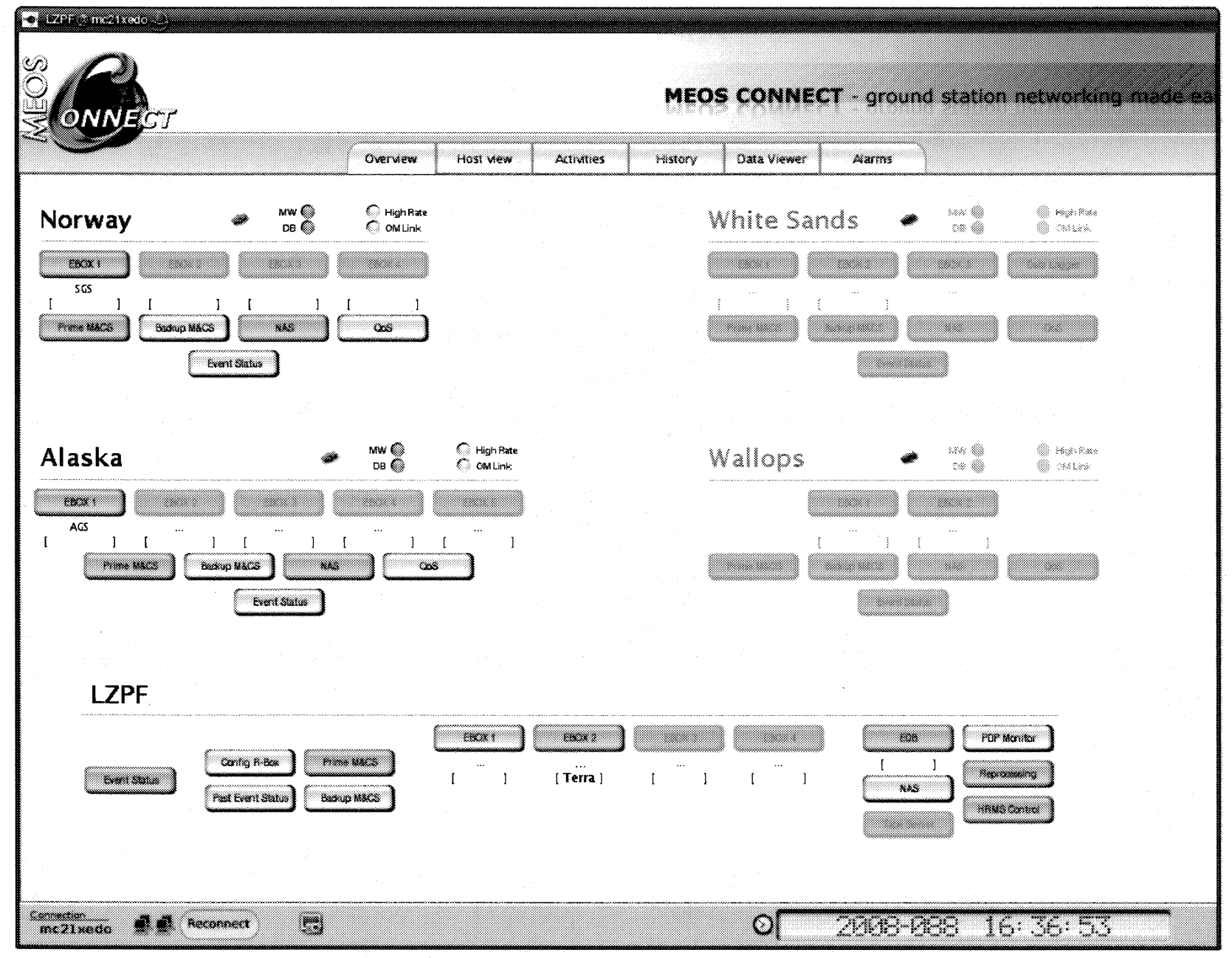

Figure 5. EDOS Main GUI Display

2. Figure 6 below is a composite view of the three Front-end Processors (FEP1, FEP2, FEP3) displaying statistics acquired during the data capture at the ground station. 


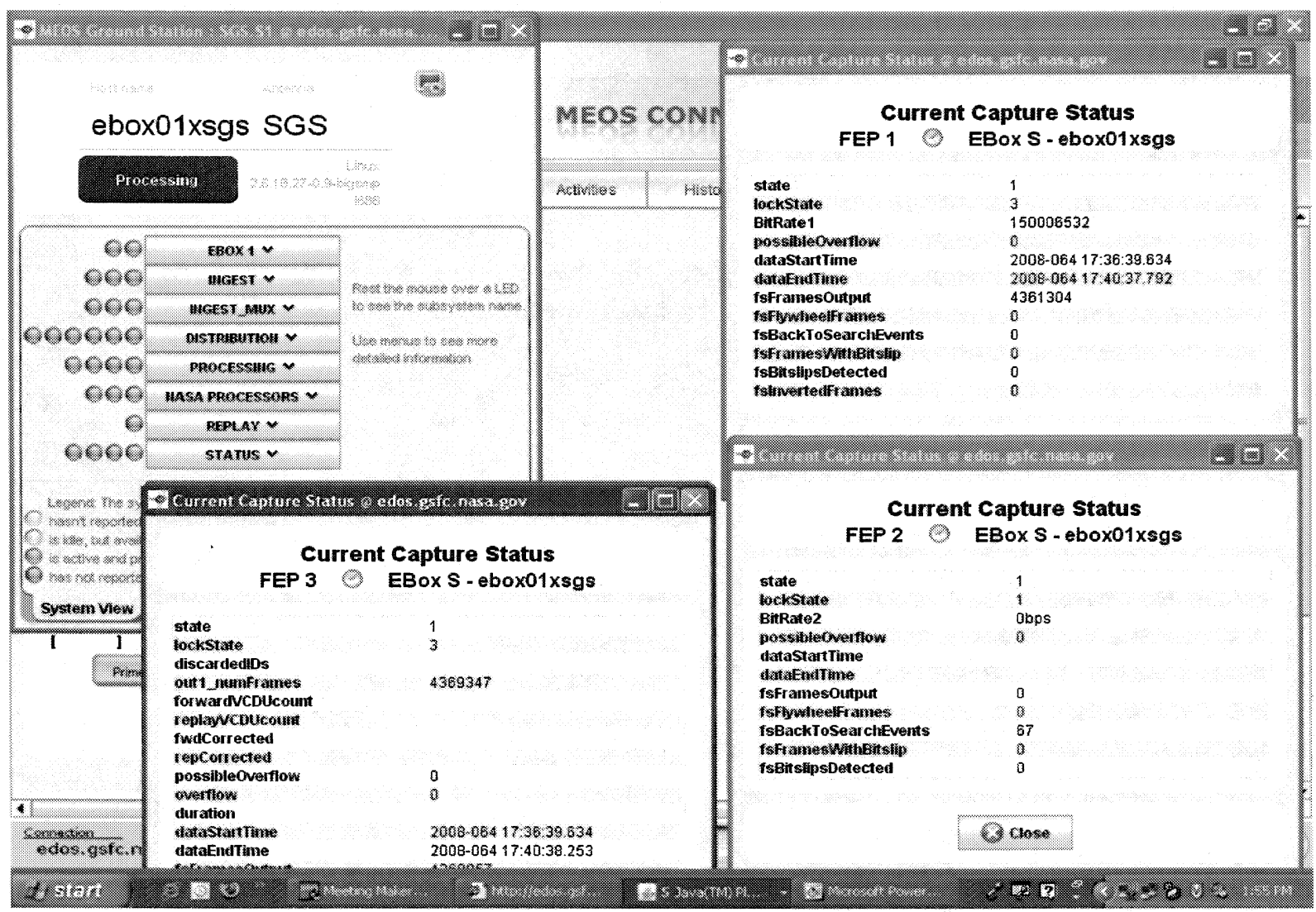

Figure 6. Front-End Processors GUI Display

3. The system view below (Figure 7) lists past events status for a given mission at an antenna site (SGS) with statistics and file transfers.

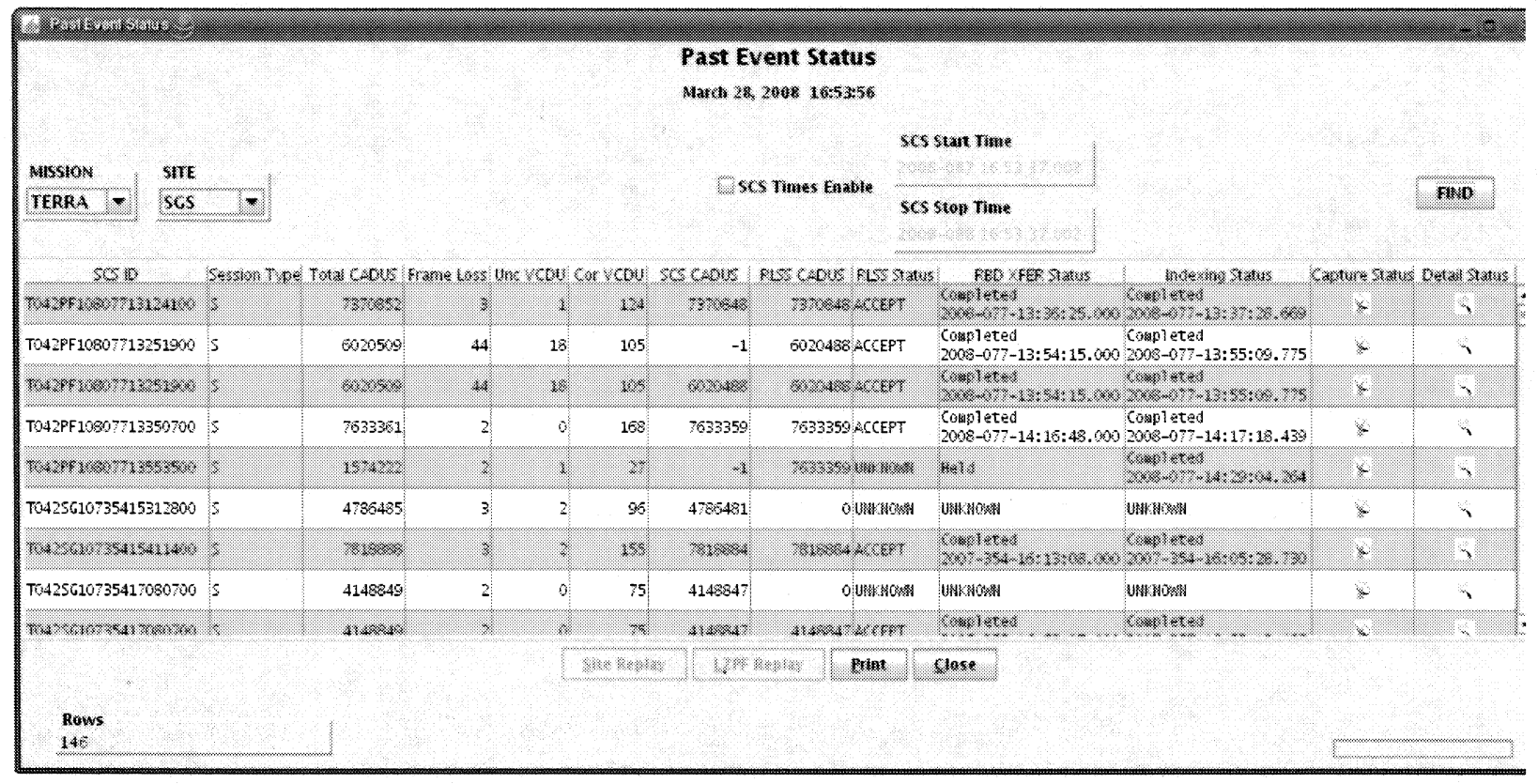

Figure 7. Front-End Processors GUI Display

American Institute of Aeronautics and Astronautics 092407 
4. The GUI view below (Figure 8) lists the Product Data Sets (PDS) created and status of the delivery to the users.

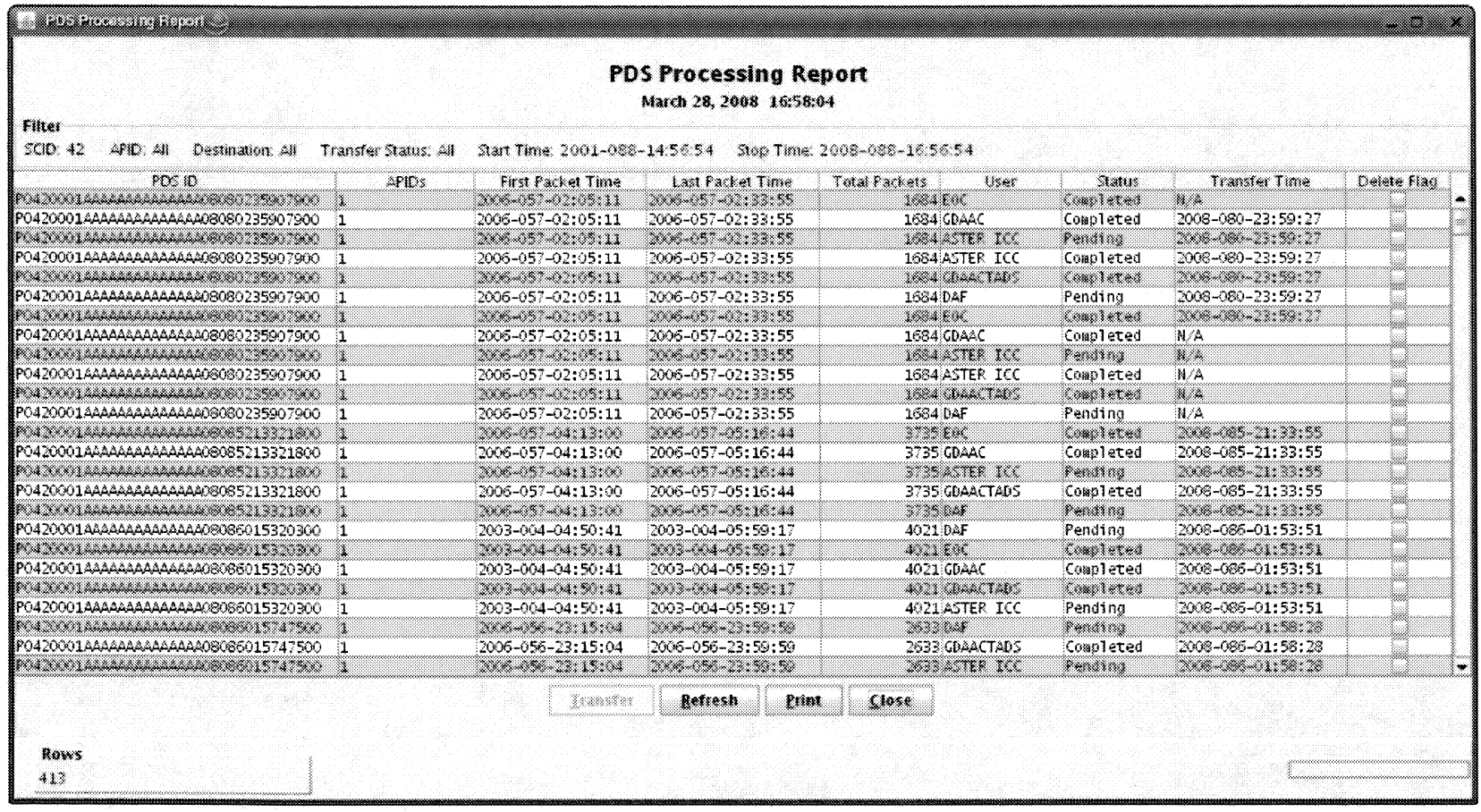

Figure 8. Display of EDOS Product Data Sets (PDS) and Delivery Status

\section{EDOS Plans}

EDOS plans to transition the new capabilities to Operations in the August 2008 time frame. This major upgrade brings in state-of-the-art technologies, the EBox- S, the EBox-R and the M\&C boxes for all sites, enabling EDOS to maintain a high level of performance in support of the current missions and expanded capabilities for support of future missions.

For later upgrades and, with respect to the M\&C System and external web monitoring, EDOS plans implementing customer profile and filtering in order to provide users with a more tailored view applicable to their mission's interests/ priorities. System anomalies detection and reporting will benefit from more applied experience via automation.

\section{Benefits / Lessons learned}

By providing web based views of the downlinks and other status information to the users, EDOS provides now instant communication/ collaboration between the ground station operators, the flight operations team and engineering mission personnel, especially when anomalies are manifesting themselves. Remote access to the information via the external web servers by users will enable a faster resolution of issues / problems and will minimize manual and operator-assisted information exchange.

Partnering with the equipment COTS provider and developer (KSPT) has proven conducive to develop the best integrated solution for monitor and control for the front-end systems in particular. Similarly the EDOS development team provides the optimum solution associated with the products generation on the EBox-R. Collaboration of this nature needs however to be well-coordinated and well-defined from the start. EDOS operations and mission engineers provided invaluable assistance in that regard by defining the content and functionality of the GUI's well in advance. 


\section{Acknowledgments}

The authors wish to express their appreciation to the NASA GSFC civil servants and contractor personnel who contributed to this program and still do. A special mention of appreciation is extended to the Mission Operations and Management Services (MOMS) EDOS support team at GSFC and to the Kongsberg Spacetec team of Tromso, Norway for their professional services and technical support.

The contractor support for this paper was provided under the Honeywell Technology Solutions Inc. (HTSI) MOMS contract \# NNG04DA01C with NASA.

\section{References}

${ }^{1}$ Cordier, G. R., Gomez-Rosa, C., Knoble, G. G., McCaleb, Fred. W., "EOS Operations Systems:

Planned and Proposed Changes to Reduce Operations Costs," Proceedings of the 6th International Symposium on Reducing the Costs of Spacecraft Ground Systems and Operations, SP-601, ESA, 2005

${ }^{2}$ Cordier, G.R., Gomez-Rosa, C., Knoble, G. G., McCaleb, Fred. W..," Autonomous multi-missions, multi-sites X-band data capture systems, " SpaceOps 2006 Conference, Rome, Italy 